\title{
Introduction to IT Architectures and Implementations in Healthcare Environments Minitrack
}

\author{
Radmila Juric \\ USN, Norway \\ rju@usn.no
}

\author{
Karen Stendal \\ USN Norway \\ Karen.Stendal@usv.no
}

\author{
Sang Suh \\ Texas A\&M University- \\ Commerce, US \\ Sang.Suh@tamuc.edu
}

The minitrack on IT Architectures and Implementation in Healthcare Environments has attracted a variety of papers, but we selected only three. They are very different in terms of problems they address in developing healthcare software applications and technologies used in their implementations.

The paper entitled "Putting Interoperability on Health Information-systems' Implementation Agenda" comes from Sweden and addresses a more than three decade old problem of heterogeneities in healthcare software solutions, which has been aggravated by the abundance of technologies we use in modern software applications, and hyper production of data we face on a daily basis. Heterogeneity is a normal characteristic of modern computing and problems of interoperability created by heterogeneity have to be addressed differently, from one specific situation to another. In this paper, the authors look at the health information systems (HIS) data exchange challenges, and propose a sense making perspective for analysing how HIS developers arrive at their interoperability design requirements. They claim that knowing "how" and knowing "which prerequisites" would enhance semantic interoperability between the different HIS is a solution. Obviously, contextual interoperability takes over as an important way of creating all round interoperability solutions.

The paper entitled "Targeting Perioperative Performance Aligned to Hospital Strategy via Digital
Transformation" come from the US from our longterm contributors. Their focus is a digital transformation of an US hospital perioperative process, which yields targeted performance alignment to strategy. The research investigates existing limitations, potential capabilities and contextual understanding in order to address the complexity of perioperative processes. Consequently, research questions on how digital

transformation can assist in targeting perioperative performance have been answered: empowered individuals, integrated IS and digitally transformed processes allow for strategic and operational performance aligned to strategy.

The paper entitled "The Care2Report System: Automated Medical Reporting as an Integrated Solution to Reduce Administrative Burden in Healthcare" comes from the Netherlands, and focuses on the integrated solution for automating the process of medical reporting. The key characteristics of their software architectural dimensions are openness and modularity, which enable solutions suitable for technical and medical software applications' perspectives. They use speech recognition technologies, linguistic annotations, semantic triples for knowledge graphs and ontologies, which in turn result in natural language generation components of the Care2Report system. 